\title{
Comment assurer la sécurité des produits de biothérapie pour les patients?
}

\author{
Hervé Brailly, ${ }^{1}$ Eric Postaire, ${ }^{2}$ Pierrette Zorzi ${ }^{3}$ et les participants à la Table Ronde $n^{\circ} 3$ \\ de Giens XIX \\ 1 Innate Pharma, Marseille, France \\ 2 INSERM-DAPS, Paris, France \\ 3 Afssaps, Saint-Denis, France
}

Résumé

\begin{abstract}
Le sujet de la table ronde étant initialement très vaste, il avait été convenu avec les participants de le recentrer sur les thérapies cellulaires (TC) impliquant des cellules autologues utilisées dans des situations de reconstruction tissulaire, d'immunomodulation et de transduction. La TC autologue concerne aussi bien des pratiques très innovantes que des produits utilisés en routine depuis plusieurs années. Le cadre réglementaire de la TC est maintenant finalisé en France. Ce dernier met en exergue le fait qu'un produit de TC est intrinsèquement lié à son procédé de préparation. L'objectif de cette table ronde était de définir les pré-requis indispensables à prendre en compte lors du développement de thérapies impliquant des préparations cellulaires ex vivo et ce, afin de s'assurer de la sécurité d'emploi de tels produits. Les différentes étapes du développement d'un produit de thérapie cellulaire (PTC) ont été envisagées : (i) contraintes de production permettant de garantir la qualité du produit; (ii) développement non clinique : importance de modèles animaux pertinents pour démontrer la preuve du concept et affiner les spécifications du PTC ; (iii) développement clinique : spécificité méthodologique du développement d'un PTC. Les problèmes inhérents à l'utilisation des produits thérapeutiques annexes (PTA) au cours du développement d'un PTC ont été soulevés. Des mesures ont été envisagées pour qu'à terme l'approvisionnement et la qualité d'un PTA ne constituent pas des facteurs limitants pour le développement de la TC en France. Enfin, une information des professionnels de santé et des industriels concernant la biovigilance s'est avérée nécessaire.
\end{abstract}

Mots clés : thérapie cellulaire, autologue, développement, pré-requis, produits thérapeutiques annexes, biovigilance

\section{Introduction}

La thérapie cellulaire (TC) autologue regroupe des techniques de préparation cellulaire à visée de reconstruction, d'immunomodulation ou de transduction d'un transgène, avec la notion commune de modification ex vivo, substantielle ou non, de la préparation cellulaire à ré-administrer. Ce champ, très large, concerne aussi bien des pratiques très innovantes mises en œuvre dans le cadre d'essais cliniques que des produits utilisés en routine depuis plusieurs années comme les préparations de cellules souches hématopoḯtiques (CSH) autologues.

La TC est un domaine particulièrement dynamique en France. A ce jour, environ 160 essais cliniques de TC et dix de thérapie génique (TG) ex vivo ont été déclarés à l'Afssaps (Agence française de sécurité sanitaire des produits de santé) [figure 1]. Plus de 90 essais de TC ont déjà été autorisés. La plus grande part de ces essais sont réalisés par des promoteurs institutionnels. Cependant, la proportion de préparation cellulaire en phase II/III est plus faible qu'aux Etats-Unis.

Aujourd'hui, le cadre réglementaire des produits de thérapie cellulaire (PTC) est finalisé en France, ce qui n'est pas encore le cas dans la plupart des pays européens. La directive 2001/83 fait entrer dans certains cas les PTC dans le champ du médicament. D'autres directives européennes sont actuellement en cours de discussion, ce qui imposera dans un avenir proche une harmonisation européenne très attendue par les professionnels impliqués dans ce domaine.

Dans le cadre de la mise en place d'essais cliniques en TC,

$\dagger$ Pour la liste des participants, voir en fin d'article. 

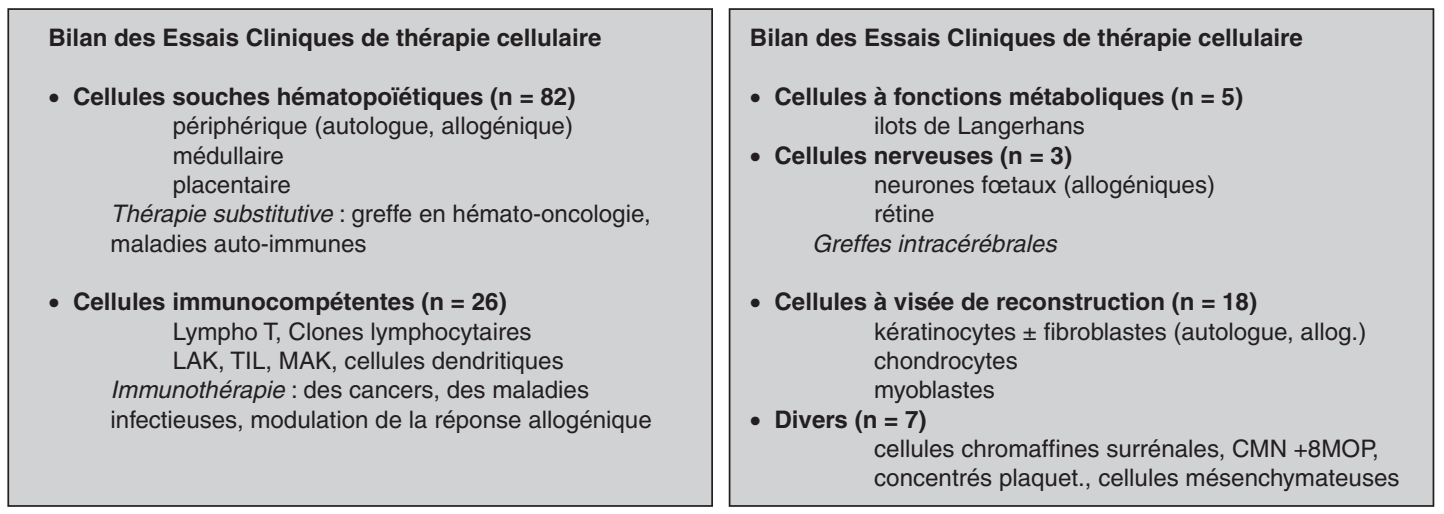

Fig. 1. Résumé de l'ensemble des essais de thérapie cellulaire soumis à l'Afssaps (Agence française de sécurité sanitaire des produits de santé) depuis 1996. $\mathbf{C M N}=$ cellules mononucléées $; \mathbf{L A K}=$ lymphocyte activated killer $; \mathbf{M A K}=$ macrophage activated killer $; \mathbf{8}$ MOP $=8$-méthoxy psoralène $; \mathbf{n}=$ nombre d'essais ; TIL = tumour infiltrating lymphocyte.

on peut percevoir une certaine dualité entre deux finalités : d'une part, les essais cliniques se situant dans une logique de développement visant à l'enregistrement du « procédé/produit » et à la production à grande échelle du PTC (dans ce cas-là, les promoteurs seront majoritairement des industriels) et, d'autre part, les essais cliniques mis en place par des médecins académiques et visant prioritairement à établir la preuve expérimentale d'un concept en thérapeutique. La difficulté du cadrage réglementaire des TC tient en partie à ce que les contraintes acceptables dans les deux cas ne sont pas exactement superposables. Il apparaît cependant clairement que l'obtention de résultats interprétables dans un essai clinique exploratoire à visée cognitive suppose la maîtrise de la reproductibilité du procédé et, bien entendu, de la sécurité. D'autres aspects du développement (notamment la propriété industrielle, la garantie d'approvisionnement en produits thérapeutiques annexes [PTA] au cours de la vie du produit) ne sont pas pris en compte de la même façon par les différents promoteurs (ceux visant à un enregistrement de leur produit/procédé et ceux réalisant des essais cliniques à visée cognitive).

L'enjeu de la cette table ronde est de dégager, pour les TC autologues, des principes généraux pour le développement s'appliquant à toutes les situations et à tous les promoteurs, et éventuellement d'identifier des questions en suspens, autour des problématiques structurant tout développement : qualité, sécurité, efficacité.

\section{Le cadre réglementaire}

La réglementation actuelle de la TC en France repose essentiellement sur trois lois :

1. la loi Bioéthique de 1994 qui introduit la notion de « préparation cellulaire »; $;^{[1]}$
2. la loi DMOSS de 1996 qui classe les produits de TC et TG dans une nouvelle catégorie de produit : les produits biologiques à effet thérapeutique ;[2]

3. et la loi du $1^{\text {er }}$ juillet 1998 qui porte sur la création de l'Afssaps. ${ }^{[3]}$

Cette dernière loi place les PTA, les organes, les tissus, les produits de TC et TG ainsi que la biovigilance sous la responsabilité de l'Afssaps.

La publication du décret 2001-909 du $1^{\text {er }}$ octobre $2001^{[4]}$ a précisé le rôle de l'Afssaps et les procédures pour la délivrance des autorisations (i) d'établissements/organismes, (ii) de procédés/produits et (iii) d'essais cliniques dans le domaine des TC et TG (figure 2). Les arrêtés du 3 février $2003,{ }^{[5-7]}$ qui finalisent ce décret, précisent les formats des dossiers à déposer auprès de l'Afssaps pour chacune de ces demandes d'autorisations.

\section{Qualité du produit de thérapie cellulaire : maîtrise du procédé}

La définition d'un PTC est intrinsèquement liée à son procédé de préparation. Un procédé de TC peut être découpé en trois étapes clés : (i) prélèvement, (ii) préparation et conditionnement et (iii) administration au patient. La maîtrise du procédé doit être assurée sur l'ensemble de la chaîne, celle des étapes finales et de la logistique afférente revêtant une importance particulière lors des essais multicentriques. La maîtrise de la qualité passe en premier lieu par le respect des principes des Bonnes Pratiques de Fabrication (BPF) au niveau du site de préparation. Elle s'applique aussi bien aux locaux, au matériel, à la formation du personnel qu'à la mise en place d'une Assurance Qualité (AQ). Cela est déjà le cas dans certaines unités pilotes en France. 
Le développement d'un PTC exige de fixer dans leurs grandes lignes les spécifications du produit, dès la réalisation des études non cliniques, quand bien même le choix de ces spécifications est amené à évoluer en raison de la compréhension très partielle que l'on a du mécanisme d'action au début du développement du PTC. En effet, le choix de ces spécifications est fonction de la destination du PTC (métabolique, reconstruction, immunologie...) et la pertinence de ces critères sera affinée au fur et à mesure du développement du PTC. Toutefois, cela ne doit pas affecter de façon substantielle les spécifications initialement définies et ces modifications ne doivent pas résulter de changements au niveau du procédé (la définition d'un PTC étant intrinsèquement liée à son procédé de préparation). Ici, se situe sans doute l'une des principales difficultés du développement des PTC, qui est celle de la pertinence des spécifications retenues, dans un contexte scientifique en constante et rapide évolution.

A titre d'exemple, les paramètres «quantité de cellules » et « viabilité » sont évidents. La définition d'un phénotype cible en fonction des effets attendus est beaucoup plus problématique. Les tests fonctionnels peuvent être très pertinents, mais extrêmement délicats à manier. Des questions scientifiques majeures quant à l'efficacité potentielle du PTC ne sont généralement pas traitées au démarrage des essais cliniques (migration des cellules, interaction avec le tissu de l'hôte...) ; la démarche de développement des biothérapies cellulaires autologues reste encore actuellement essentiellement empirique.

Selon que ces produits seront des spécialités pharmaceutiques ou des non-spécialités pharmaceutiques, les mêmes exigences de qualité seront requises.

\section{Problématique des produits de thérapeutique annexe}

On entend par PTA « tout produit, à l'exception des dispositifs médicaux mentionnés à l'article L.5211-1, entrant en contact avec des organes, tissus, cellules, ou produits issus du corps humain ou d'origine animale au cours de leur conservation, de leur préparation, de leur transformation, de leur conditionnement ou de leur transport avant leur utilisation thérapeutique chez l'homme, ainsi que tout produit entrant en contact avec des embryons dans le cadre d'une activité d'assistance médicale à la procréation ».

Ainsi, tous les produits utilisés au cours d'un procédé de TC répondent à la définition de PTA. Ces derniers concernent une large gamme de produits, de simples à très complexes.

Dès la parution du décret sur les PTA, les fabricants souhaitant obtenir une autorisation de mise sur le marché (AMM) pour un nouveau PTA devront en faire la demande auprès de l'Afssaps. Le dossier fourni à l'appui de cette demande, moins contraignant que celui utilisé pour tout nouveau médicament, devra néanmoins garantir des niveaux satisfaisants de sécurité, qualité et efficacité in vitro du PTA.

Dès lors, les professionnels de la TC pourront faire référence dans leur dossier de demande d'autorisation de procédé/produit à l'autorisation PTA délivrée par l'Afssaps. Dans le cas où le produit n'a pas encore le statut PTA (ou bien s'il est en cours d'évaluation par l'Afssaps), il appartient au responsable de la demande d'autorisation d'un PTC de documenter pour les PTA qu'il utilise, l'innocuité, la qualité et l'efficacité in vitro revendiquée. Enfin, si le PTA dispose déjà d'une autre autorisa-
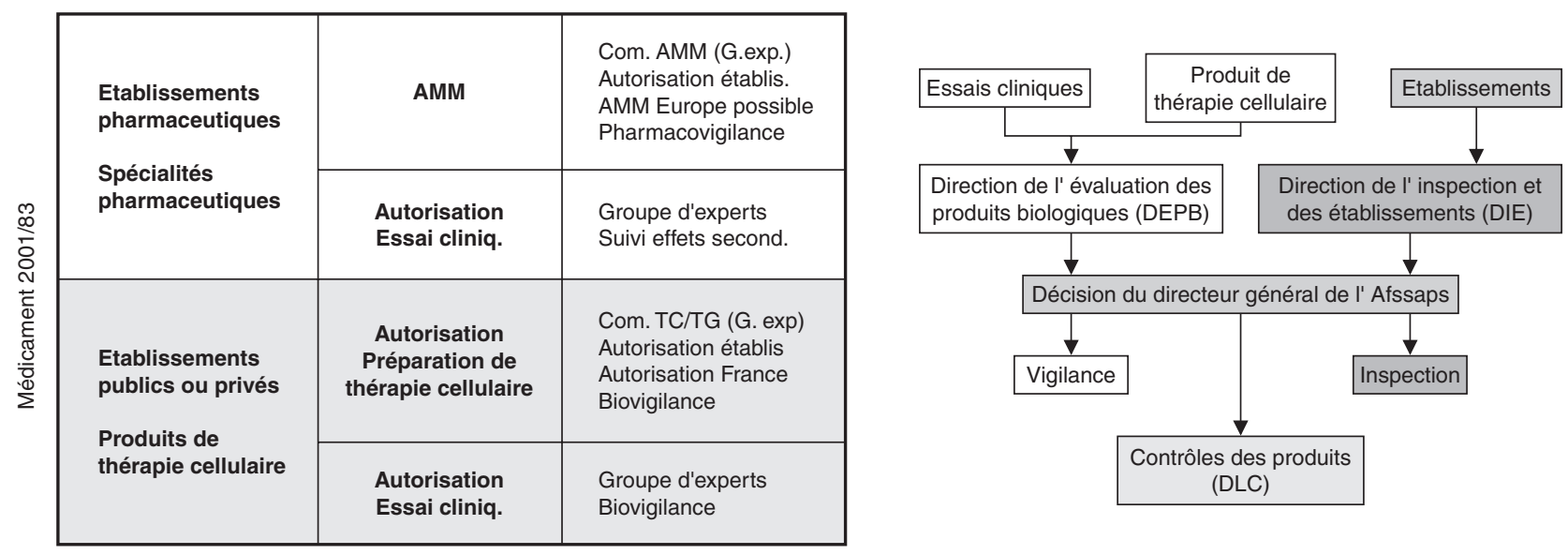

Fig. 2. Procédures mises en place par l'Afssaps (Agence française de sécurité sanitaire des produits de santé) selon que les préparations cellulaires seront des spécialités pharmaceutiques ou des non-spécialités pharmaceutiques. AMM = autorisation de mise sur le marché ; DLC = Direction des laboratoires et des contrôles ; G exp. = groupe d'experts ; TC = thérapie cellulaire ; TG = thérapie génique. 
tion délivrée par l'Afssaps (médicament), la référence à la dite autorisation sera suffisante.

Compte tenu de la mise en place progressive de ce statut réglementaire, les professionnels de la TC ne disposeront pas avant plusieurs années d'une liste officielle de PTA autorisés. Ici se pose le problème majeur de la disponibilité des PTA présentant des garanties de qualité, d'innocuité et d'efficacité. La TC n'est pas constituée en marché mature susceptible d'intéresser un industriel à une démarche de développement spécifique de PTA, alors que la possibilité de mener à terme certains essais peut dépendre de l'approvisionnement en certains facteurs de croissance. Une première étape de recensement des besoins en PTA auprès des promoteurs d'essais cliniques de TC serait indispensable. Dans un second temps, une mutualisation de la production de certaines biomolécules devra être mise en place, à moins que la maturation du marché incite des opérateurs spécialisés à prendre position. D'une façon générale, il est recommandé de ne s'engager dans un essai clinique de PTC que si la maîtrise de l'approvisionnement en PTA de qualité peut être assurée, ces PTA devant être préparés dans des conditions de BPF.

\section{Développement non clinique : preuve du concept}

Une distinction fondamentale peut être faite du point de vue des preuves préliminaires d'efficacité entre les TC utilisées en immunothérapie, principalement en oncologie, et les TC à visée de reconstruction tissulaire ou de correction d'un déficit génétique. En immunothérapie, on se heurte d'emblée à des problèmes de transposabilité des modèles animaux disponibles, en particulier dans les situations de vaccination thérapeutique (par exemple cellules dendritiques chargées), plus encore que dans les cas d'administration de cellules effectrices (macrophages, lymphocytes cytotoxiques...). Pour les essais cliniques d'immunothérapie adoptive, les systèmes cellulaires sont suffisamment conservés, de sorte que des modèles syngéniques peuvent être pertinents. Au contraire, les différences inter-espèces s'opposent à des évaluations pertinentes de l'immunogénicité de préparations cellulaires à finalité de vaccination thérapeutique, visant à déclencher une réponse immune chez l'hôte. Des progrès significatifs pourront sans doute résulter de l'émergence de nouveaux modèles d'animaux transgéniques permettant d'évaluer l'immunogénicité dans un contexte de CMH humain. Pour les situations de reconstruction tissulaire, on dispose au contraire de l'immunothérapie d'un grand nombre de modèles pertinents qui peuvent et doivent être utilisés en préclinique (notamment hépatologie, cardiologie, myopathies).
Quel que soit le contexte thérapeutique étudié, les modèles animaux jouent un rôle important notamment dans l'exploration de la voie d'administration du PTC, et ils doivent être systématiquement envisagés.

\section{Développement non clinique : toxicologie}

Les risques à évaluer sont les mêmes, qu'il s'agisse d'un PTC ou d'un produit conventionnel : toxicité lors de l'administration unique ou réitérée, identification des organes cibles, toxicité sur les fonctions de reproduction, pouvoir mutagène et cancérogène, tolérance locale et immunotoxicité. Ces questions sont très cadrées par les dispositions réglementaires en vigueur (CPMP [Commission d'AMM des médicaments], ICH [International Committee on Harmonisation]). Cependant, lors de la conception du plan de développement, les modalités employées pour répondre à ces questions doivent prendre en compte les spécificités des PTC, et notamment la disponibilité de modèles animaux pertinents. Ces études doivent être réalisées avec le PTC qui fera l'objet de l'essai clinique. Certains points particuliers sont à prendre en compte : (i) la recherche des organes cibles de la toxicité doit se faire avec un modèle animal pertinent au regard de la situation clinique envisagée, notamment pour ce qui concerne la voie d'administration, l'utilisation éventuelle d'implant, etc. ; (ii) lorsque les cellules autologues ré-injectées produisent une molécule biologiquement active (cytokine, facteur de croissance), la toxicité du produit sécrété doit être documentée ; et (iii) le risque de cancérogénèse doit être soigneusement documenté en utilisant plusieurs lots successifs de PTC dans le cas d'emploi de matériel cellulaire susceptible de proliférer après ré-injection.

On retrouve en toxicologie la problématique précédemment évoquée, à savoir la nécessité d'une définition suffisante des spécifications du produit. L'absence de modèle et l'évolutivité des spécifications rendent encore plus nécessaire que dans le cas de produits conventionnels l'établissement d'un dialogue précoce avec les agences.

\section{Développement clinique}

La question principale posée était celle des spécificités méthodologiques du développement clinique des PTC, en particulier pour ce qui concerne le découpage des phases exploratoires précoces en phase I dédiée à l'évaluation de la tolérance, et phase II consacrée à la preuve du concept. Ce découpage fait débat, notamment pour des indications orphelines sans alternative thérapeutique, qui constituent autant de cas d'espèce à discuter avec les agences, et compte tenu des difficultés qu'il peut y avoir à définir une dose maximum tolérée (DMT) en phase I. Cepen- 
dant, en immunothérapie antitumorale, compte tenu des informations non cliniques parcellaires dont on dispose, il semble préférable de mener une véritable phase I visant en objectif principal à évaluer la sécurité d'emploi du produit. Bien entendu, des données biologiques pertinentes peuvent être collectées en phase I par un immunomonitoring adapté. Les problèmes posés par la phase II sont alors ceux, plus généraux, des essais réalisés sur des effectifs faibles et hétérogènes, notamment pour ce qui concerne la définition du groupe contrôle. L'immunothérapie anticancéreuse pose des problèmes particuliers, dans la mesure où l'on s'adresse en première intention à des patients à des stades avancés, éventuellement associés à une immunosuppression systémique. Cette population n'est pas nécessairement représentative du groupe de patients ciblés par le PTC. Cette contrainte souligne l'importance d'une bonne définition immunologique des populations testées, sans que soit remise en cause la méthodologie générale du développement clinique. La phase III, quant à elle, reste la démonstration statistique nécessairement multicentrique de l'efficacité clinique du PTC. La phase III pose des problèmes particuliers de maîtrise de la reproductibilité du procédé de TC et de distribution des lots de PTC entre les différents sites.

Dans un contexte de développement global, il semble que des réponses plus satisfaisantes pourraient être apportées dans le cadre d'une centralisation du traitement des PTC dans un site unique, pour un procédé donné.

\section{Pharmacovigilance/biovigilance}

La parution prochaine du décret de biovigilance viendra compléter le dispositif réglementaire qui existe déjà en matière de vigilance pour les autres produits de santé (pharmacovigilance, matériovigilance, réactovigilance...). Un PTC utilisé dans le cadre d'un essai clinique ou dans le cadre d'une activité de routine ainsi que tous les PTA utilisés au cours de ces procédés entreront dans le champ de compétence de la biovigilance.

Par contre, les PTC qui auront obtenu le statut de spécialité pharmaceutique entreront dans le champ de compétence de la pharmacovigilance.

Des critiques ont été formulées par le groupe sur la mise en œuvre de ce nouveau dispositif. Ces critiques portent principalement sur l'apparente complexité du système et sur les risques afférents à une mauvaise appropriation par les acteurs industriels (un PTC pouvant avoir ou non le statut de spécialité pharmaceutique, un PTA pouvant avoir le statut de spécialité pharmaceutique). Il est toutefois reconnu que les PTC posent des problèmes spécifiques non adressés par le système existant de la pharmacovigilance, qui justifie de la mise en place d'un système dédié.

Pour autant, le groupe recommande que la mise en œuvre soit rapidement l'objet d'une concertation avec les industriels et les professionnels de la TC.

\section{Recommandations/actions}

Les recommandations générales vont dans le sens d'une meilleure prise en compte de la logique du développement et de la maîtrise de la qualité des PTC dans les essais institutionnels, afin d'améliorer la qualité des données cliniques et leur interprétation, et de faciliter le transfert vers l'industrie au niveau des phases tardives de développement.

- Mise aux normes BPF des sites de production.

- Moyens accrus pour soutenir les protocoles issus du secteur académique (affaires réglementaires, AQ...).

- Dialogue précoce des développeurs avec l'Afssaps.

Par ailleurs, le groupe de travail a identifié un certain nombre de facteurs limitants affectant de manière récurrente tous les opérateurs (harmonisation européenne de la réglementation, disponibilité des PTA, mise en application du décret biovigilance). Aussi, les actions suivantes sont proposées :

- recensement des PTA ;

- en fonction de ce recensement, études de faisabilité de la production mutualisée (européenne ?) de PTA, selon le modèle RAID (Rapid Access to Intervention Development) du NCI (National Cancer Institute) ;

- actions visant à l'harmonisation réglementaire européenne, autour de la transposition pour les PTC de la directive 2001/83;

- mise en place d'un groupe de travail « biovigilance » impliquant les industriels.

\section{Participants}

L. Becquemont (Pharmacologie, CHU du Kremlin Bicêtre), F. Bassompierre (DRC, AP-HP), B. Dreno (Inserm U463, Nantes), S. Lucas-Samuel (Afssaps, Remerciements à K. Martinière, Afssaps), M. Pallardy (Inserm U461, Châtenay Malabry), S. Scholl (Institut Curie, Paris), M. Bonneville (Inserm, Nantes), P. Zorzi (Afssaps), E. Postaire (Coordonnateur, INSERMDAPS Paris), P.-Y. Arnoux (Leem), I. Barilero (Johnson \& Johsnon), E. Caugant (Wyeth, Paris), D. Debiais (Amgen, Neuilly/seine), M. Gersberg (Sanofi, Le Plessis Robinson), J. Tiollier (Innate Pharma, Marseille), H. Brailly (Innate Pharma, Marseille), Y. Kabrane (Afssaps), J.-L. RometLemonne (IDM), P. Squiban (Transgène), S. Solbès Latourette (AFM, Evry).

\section{Références}

1. Loi n ${ }^{\circ}$ 94-654 du 29 juillet 1994 relative au don et à l'utilisation des éléments et des produits du corps humain, à l'assistance médicale à la procréation et au diagnostic prénatal [online]. Available from URL: http://www.legifrance.gouv.fr/WAspad/UnTexteDeJorf?.numjo=SPSX9400032L [Accessed 2004 Jul 19] 
2. Loi n ${ }^{\circ} 96-452$ du 28 mai 1996 portant sur diverses mesures d'ordre sanitaire, social et statutaire [online]. Available from URL: http://www.social.gouv.fr/htm/ minister/igas/igas_loi3.htm [Accessed 2004 Jul 19]

3. Loi $\mathrm{n}^{\circ}$ 98-535 du 1er juillet 1998 , relative au renforcement de la veille sanitaire et du contrôle de la sécurité sanitaire des produits destinés à l'homme [online]. Available from URL: http://www.legifrance.gouv.fr/WAspad/UnTexte DeJorf?.numjo=MESX9702267L [Accessed 2004 Jul 19]

4. Décret $n^{\circ} 2001-909$ du $1^{\text {er }}$ octobre 2001 relatif aux cellules et aux produits de thérapies génique et cellulaire fixant les conditions d'autorisation des établissements, organismes, procédés, produits et protocoles d'essais cliniques et modifiant le code de la santé publique [online]. Available from URL: http://www.arteb.com/infos/LoiDecrets/decret2001-909.pdf [Accessed 2004 Jul 19]

5. Arrêté du 3 février 2003 fixant le contenu du dossier accompagnant la demande d'autorisation des procédés de préparation, de conservation et de transformation des cellules et des produits de thérapie cellulaire [online]. Available from URL: http://perso.club-internet.fr/dominique.mathis/bdlr/arr2000/ A030203h. htm [Accessed 2004 Jul 19]
6. Arrêté du 3 février 2003 fixant le contenu des dossiers d'autorisation, de renouvellement et de modification de l'autorisation des établissements ou organismes exerçant des activités de préparation, de conservation, de distribution et de cession de cellules et de produits de thérapies génique et cellulaire qui ne constituent pas des spécialités pharmaceutiques ou d'autres médicaments fabriqués industriellement [online]. Available from URL: http://perso.clubnternet.fr/dominique.mathis/bdlr/arr2000/A030203g.htm [Accessed $2004 \mathrm{Jul}$ 19]

7. Arrêté du 3 février 2003 relatif aux conditions d'autorisation pour la mise en œuvre des protocoles d'essais cliniques portant sur des cellules issues du corps humain, des produits de thérapie cellulaire et des produits de thérapie génique [online]. Available from URL: http://perso.club-internet.fr/dominique.mathis/ bdlr/arr2000/A030203f.htm [Accessed 2004 Jul 19]

Correspondance et offprints : Eric Postaire, INSERM-DAPS, 101 rue de Tolbiac, 75654 Paris Cedex 13, France.

E-mail : postaire@tolbiac.inserm.fr 